\title{
$63^{\text {rd }}$ International Congress and Annual Meeting of the Society for Medicinal Plant and Natural Product Research (GA2015) \\ Budapest, Hungary, 23 - 27 August 2015
}

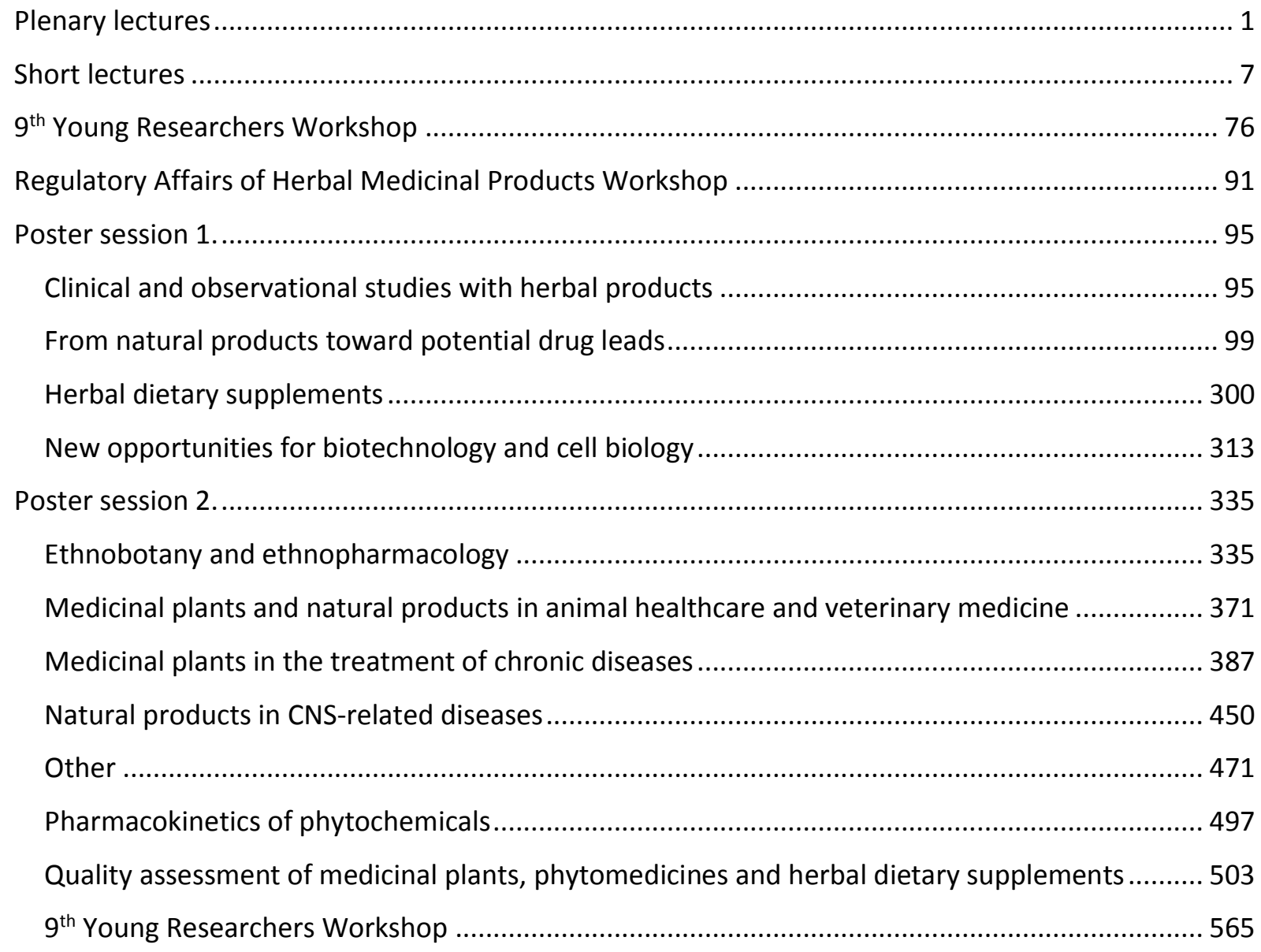


PM-189

\section{Caffeic acid derivates in spent coffee ground as potential crude material for drug} discovery

Vanessa Mendes ${ }^{1}$, Sónia Mendes ${ }^{1}$, Bárbara Gomes ${ }^{1}$, Ofélia Anjos $^{2,3}$, Maria Graça Campos ${ }^{1}$

${ }^{1}$ Drug Discovery Group, Centre for Pharmaceutical Studies, Faculty of Pharmacy, University of Coimbra, Health Sciences Campus, Azinhaga de Santa Comba,, 3000 - 548 Coimbra, Portugal

${ }^{2}$ Instituto Politécnico de Castelo Branco, Escola Superior Agrária, Apartado 119,, 6001 - 909 Castelo Branco, Portugal

${ }^{3}$ Centro de Estudos Florestais, Instituto Superior de Agronomia, Universidade de Lisboa, Tapada da Ajuda, 1349 - 017 Lisboa, Portugal

The aim of this work is to evaluate the methodology to obtain an extract rich in caffeic acid (CAD) derivatives in spent coffee ground (SCG) and evaluate its free radical scavenging activity. Eighteen SCG samples and coffee beverage were collected from different brands and coffee shops. The SCG extracts were prepared by three methods: A) ethanol 96o according Campos et al [1]; B) extracts prepared in A were hydrolysed with $\mathrm{HCl} \mathrm{37 \%} \mathrm{(1:10),} \mathrm{according}$ Markham [2]; C) water extraction. All samples were screened by HPLC/DAD for phenolic profile characterization and structural identification and quantification of CAD [1]. Among caffeine the majority of the compounds found in the coffee beverage were CAD and in the SCG the compounds remaining the same. The hydrolysed extracts confirmed its existence, possible as glycosides (Fig 1). The matrix resulting from the hydrolysis of the SCG can also be used to explore further bioactivities for therapeutic consideration once the released aglycones are in general more active. Method A was the most efficient (4.305 mg CAD/g SCG). Nevertheless the results were different according to the products. The caffeine ratio between SCG and Expresso coffee was $17 \%$ and the ratio of CAD in both samples was $8 \%$. A significant free radical scavenging potential (activity of DPPH) was founded yet in SCG $\left(\mathrm{EC}_{50}=1.857 \mu \mathrm{g} / \mathrm{mL}\right)$ comparing with the coffee beverage $\left(\mathrm{EC}_{50}=0.172 \mu \mathrm{g} / \mathrm{mL}\right)$ around $10 \%$ lower. The results show that the SCG has potential for future biological screening showing a stable compound profile and constitutes a much simpler matrix than the beverage, simplifying its analysis and further studies for potential bioactivities.

[1] Campos MG, Mitchel K, Cunha A, Markham K., A systematic Approach to the characterisation of Bee Pollens via their Flavonoid/Phenolic Profiles. Phytochem. Anal. 1997; 8:181-185. 2. Markham KR., Techniques of Flavonoid Identification. Academic Press, London; 1982: 52-53. 


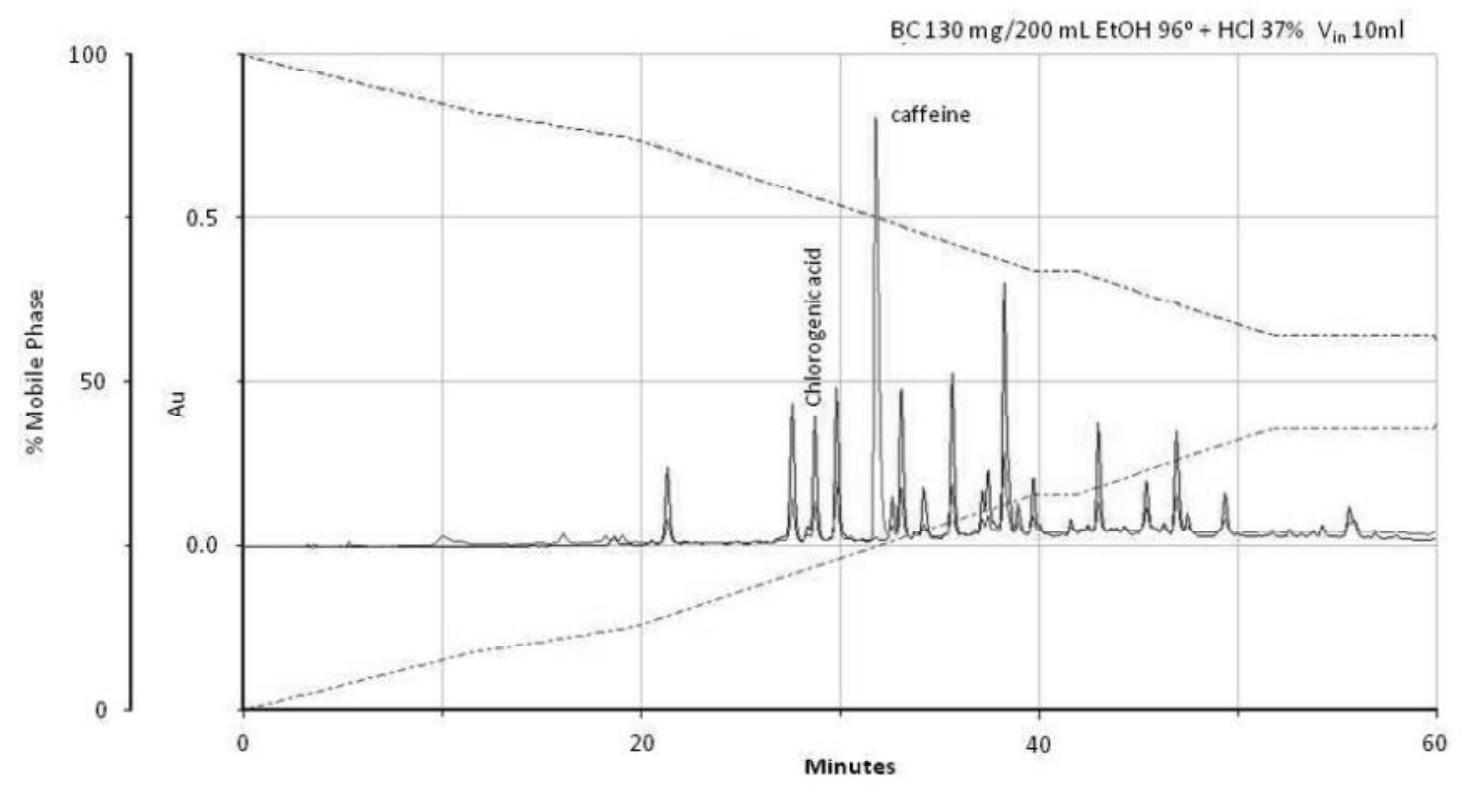

Figure 1. HPLC/DAD profile of acidic hydrolyse of ethanolic extract recorded at $\lambda_{\max } 260$ (for caffeine)(green line) and $340 \mathrm{~nm}$ (for caffeic acid derivatives)(red line).

PM-190

Protective effect and mechanism of triterpenoids isolated from Cornus walteri against anticancer drug-induced nephrotoxicity in LLC-PK1 cells

Jae Sik Yu, Seulah Lee, Hee Jeong Eom, Hee Rae Kang, Seoung Rak Lee, Ki Hyun Kim

Natural Product Research Laboratory, School of Pharmacy, Sungkyunkwan University, Suwon, Korea, Republic of (South)

Cornus walteri Wanger (Cornaceae) is a deciduous shrub distributed in valley areas of Asia, and its fruits and leaves have been used in Chinese folk medicine for treatment of inflammation of the skin or boils caused by lacquer poison [1]. In our search for bioactive constituents from Korean medicinal plants, a phytochemical investigation of the $\mathrm{MeOH}$ extract of the stems and stem bark of $C$. walteri resulted in identifying twelve major triterpenoids (1-12). Compounds 1-4 ameliorated cisplatin-induced nephrotoxicity to $80 \%$ of the control value at $125 \mu \mathrm{M}$. Phosphorylation of mitogen-activated protein kinases (MAPKs) was decreased following cisplatin treatment after treatment with compounds 1-4. These results show that blocking the MAPKs signaling cascade plays a critical role in mediating the renoprotective effect of the isolated compounds1-4 from the stems and stem bark of $C$. walteri. 